\title{
Soft Microscopy: Strategies for Contrast Enhancement of Macromolecules
}

Kelly Parker ${ }^{1}$, Shengwang Zhou ${ }^{1}$, Blaise Kimmel ${ }^{1}$, Sonali Dhindwal ${ }^{1}$, Reiner Bleher ${ }^{1}$, Cheri Hampton ${ }^{2}$, Lawrence Drummy ${ }^{2}$, Milan Mrksich ${ }^{1}$ and Vinayak Dravid ${ }^{1}$

${ }^{1}$ Northwestern University, Evanston, Illinois, United States, ${ }^{2}$ Air Force Research Laboratory, Dayton, Ohio, United States

The low inherent contrast of soft systems necessitates contrast enhancement by either sample preparation or imaging methodologies not often employed during hard material imaging. This improved contrast is commonly achieved through either positive or negative heavy metal staining, which obscures fine features due to the grain size of the stain. We have used a number of these methods to enhance contrast, but we also aim to extract the contrast inherent in soft systems by manipulating microscope conditions. Lowvoltage electron microscopy enables contrast enhancement by increasing the electron scattering crosssection, with the trade-off of slight resolution degradation due to increased chromatic aberration [1]. This allows for the imaging of unstained soft materials such as precisely-defined protein constructs - known as megamolecules - that are synthesized from fusion protein building blocks into macromolecular assemblies [2]. 3-dimensional reconstruction methods are then possible to observe the spatial arrangement of individual proteins within the larger construct. Here, we use transmission electron microscopy (TEM) as well as scanning electron microscopy (SEM) with scanning TEM (STEM) detection (SEM/STEM) to observe the structures of stained and unstained megamolecules.

The tetracutinase megamolecule (shown schematically in Figure 1a) was synthesized by the reaction of the cutinase enzyme with a four-armed linker, generating a $95 \mathrm{kDa}$ tetramer product. The protein constructs were first dropcast onto hydrophilized carbon-film TEM grids and stained with $0.75 \%(\mathrm{w} / \mathrm{v}$ ) uranyl formate, and imaged with $200 \mathrm{kV}$ TEM and STEM. In order to obtain class averages and improve contrast, reference-free 2D classification of the stained structures was performed using the Relion software [3]. To obtain images at voltages lower than is possible in classical TEM or dedicated STEM, the megamolecules were imaged using SEM/STEM from $5 \mathrm{kV}-30 \mathrm{kV}$. Unstained tetracutinase megamolecules were deposited on a graphene oxide thin film and imaged using $40 \mathrm{kV}$ TEM. The distances between cutinase proteins were compared with those determined by X-ray crystallography.

Figure 1 shows tetracutinase stained with uranyl formate and imaged under various voltages in STEM, from $200 \mathrm{kV}$ down to $5 \mathrm{kV}$. Figure 1a was imaged in a dedicated STEM in high angle annular dark field (HAADF) mode, while Figures 1b-f were imaged in SEM/STEM in order to obtain voltages lower than possible in conventional TEM. The insets in each image show a magnified single megamolecule at similar orientations, in order to compare contrast and resolution. The protein lobes were resolvable in both bright field (BF) and HAADF STEM at voltages as low as $5 \mathrm{kV}$, although resolution deteriorates at lower kV because of increased chromatic aberration. Though reasonable contrast is achieved at low voltages in SEM/STEM, sufficient contrast and improved resolution is obtained at $200 \mathrm{kV}$ in a dedicated STEM due to the contrast provided by the heavy metal stain. However, this method of staining obscures fine features of the protein constructs by introducing stretching and blurring of individual proteins and does not offer an accurate representation for size measurements and other analysis.

Cryo-electron microscopy is a widely used technique for protein imaging, but it is lower-throughput than room temperature imaging for the structure characterization of a diverse set of megamolecules [4]. Lowvoltage electron microscopy, on the other hand, can be used to achieve sufficient contrast of unstained 
proteins. Tetracutinase was deposited and dried on thin graphene oxide, which is hydrophilic to allow for protein deposition and is thin enough to mitigate chromatic aberration and beam broadening through the film. Figure 2 a shows a micrograph imaged in $40 \mathrm{kV}$ TEM with multiple tetracutinase megamolecules oriented appropriately to observe the four lobes. The distances between lobes were compared with those determined by X-ray crystallography, as shown in Figure $2 \mathrm{~b}-\mathrm{c}$, and match to within $0.5 \mathrm{~nm}$. When compared with a class-averaged image with a similar orientation from negative-stained tetracutinase (Figure 2d), which blurs features significantly, unstained low-kV imaging provides significantly more accurate protein spacing and eliminates the stretching of features from negative staining. Though the resolution is not comparable to that obtained by cryo-EM and single particle reconstruction, the goal of this effort is not to solve individual protein structures, but rather to observe the architecture of linked proteins. Low-kV TEM of unstained proteins provides sufficient resolution for this objective.

This work demonstrates the application of low-kV electron microscopy to protein megamolecules, as observing their structure is essential to understanding their function as large macromolecular building blocks. The combination of low accelerating voltage and ultrathin substrates provides the contrast necessary to visualize these and other soft structures, while minimizing the effect of chromatic aberration on resolution. Further work will be done in imaging unstained soft materials by SEM/STEM to reduce voltage to SEM levels, while also reaping the benefits of STEM imaging and mass-thickness contrast for applications such as molecular weight determination. Gentler drying methods, such as freeze drying, can be used to further reduce structural alterations. We also plan to perform class averaging and single particle reconstruction of unstained structures on thin films in order to understand the configuration of the protein lobes in 3-dimensional space, comparing with architectures determined by cryo-EM. This method can be used to observe the structure of more complex megamolecules and other soft constructs without the detrimental effects to spatial resolution from negative staining, while maintaining the high-throughput nature of room temperature imaging [5].
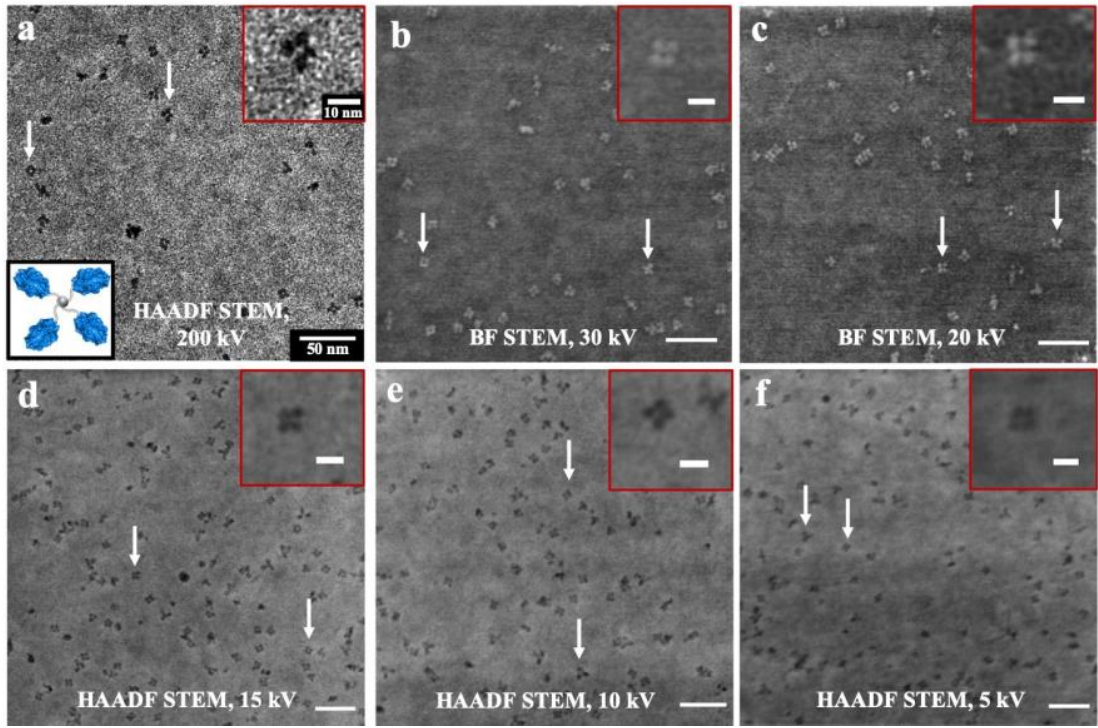

Figure 1. STEM images of uranyl formate-stained tetracutinase with magnified insets a) at $200 \mathrm{kV}$ on a dedicated STEM, b-f) at $30 \mathrm{kV}, 20 \mathrm{kV}, 15 \mathrm{kV}, 10 \mathrm{kV}$ and $5 \mathrm{kV}$, respectively, on an SEM with a STEM detector. 


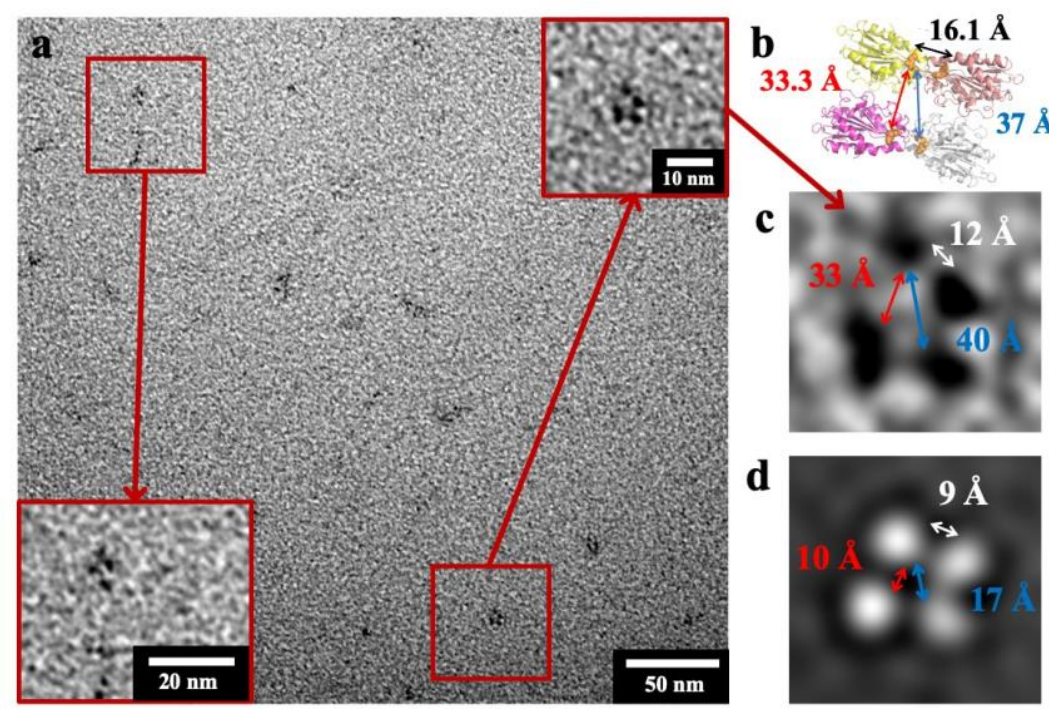

Figure 2. a) TEM image of unstained tetracutinase on a graphene oxide film at $40 \mathrm{kV}$. b) Tetracutinase structure determined by X-ray crystallography with distances between proteins labeled. c) Magnified image of unstained tetracutinase with corresponding inter-protein distances labeled. d) Class-averaged image of uranyl formate-stained tetracutinase in a similar orientation.

\section{References}

[1] Nixon, W. C. Physikalisch-Technischer Teil (1960), p. 302-306.

[2] Modica, J. A., Skarpathiotis, S. \& Mrksich, M. ChemBioChem 13 (2012), p. 2331-2334.

[3] Scheres, S. H. W. J. Struct. Biol. 180 (2012), p. 519-530.

[4] Grassucci, R. A., Taylor, D. J., \& Frank, J. Nature Protocols 2 (2007), p. 3239-3246.

[5] This work was supported by Air Force Research Laboratory grant FA8650-15-2-5518, Army Research Office grant W911NF1810200, and the National Science Foundation Graduate Research Fellowship Program. This work made use of the Electron Probe Instrumentation Center and BioCryo facilities of Northwestern University's NUANCE Center, which has received support from the Soft and Hybrid Nanotechnology Experimental (SHyNE) Resource (NSF ECCS-1542205); the MRSEC program (NSF DMR-1720139) at the Materials Research Center; the International Institute for Nanotechnology (IIN); the Keck Foundation; and the State of Illinois, through the IIN. 